British Journal of Nutrition (2022), 127, 257-265

doi:10.1017/S0007114521000970

(C) The Author(s), 2021. Published by Cambridge University Press on behalf of The Nutrition Society. This is an Open Access article, distributed under the terms of the Creative Commons Attribution-NonCommercial-NoDerivatives licence (http://creativecommons.org/licenses/by-nc-nd/ 4.0/), which permits non-commercial re-use, distribution, and reproduction in any medium, provided the original work is unaltered and is properly cited. The written permission of Cambridge University Press must be obtained for commercial re-use or in order to create a derivative work.

\title{
Adiponectin and 8-epi-PGF $2 \alpha$ as intermediate influencing factors in weight reduction after legume consumption: a 12-week randomised controlled trial
}

\author{
Youngmin $\mathrm{Han}^{1}$, A Ram Kim², Jong Ho Lee ${ }^{1}$ and Minjoo Kim* \\ ${ }^{1}$ National Leading Research Laboratory of Clinical Nutrigenetics/Nutrigenomics, Department of Food and Nutrition, College of \\ Human Ecology, Yonsei University, Seoul, South Korea \\ ${ }^{2}$ Department of Science for Aging, Graduate School of Yonsei University, Seoul, South Korea \\ ${ }^{3}$ Department of Food and Nutrition, College of Life Science and Nano Technology, Hannam University, Daejeon, South Korea
}

(Submitted 13 August 2020 - Final revision received 22 February 2021 - Accepted 8 March 2021 - First published online 22 March 2021)

\section{Abstract}

Legumes are rich sources of essential nutrients, and their potential health benefits were reported in many studies. Several studies showed a positive effect of legumes on obesity, but randomised clinical trials are limited in the Korean population. The present intervention study investigated the impact of legumes on body weight in obese Korean subjects. A total of 400 participants (BMI $\geq 25 \mathrm{~kg} / \mathrm{m}^{2}$ ) were randomised into two groups. The legume-enriched diet (LD) group replaced one-third of their refined rice consumption with legumes three times per day as a carbohydrate source. In contrast, the usual diet (UD) group consumed their UD. The mean weight loss at 12 weeks was 2.87 (SEM 0.21$) \mathrm{kg}$ and 0.17 (SEM 0.11$) \mathrm{kg}$ in the LD and UD, respectively, which was significantly different between the groups $(P<0.001$ ). HDL-cholesterol and adiponectin levels were increased, and levels of glucose, insulin, TAG, and 8-epi-PGF $2 \alpha$ and the homoeostasis model assessment of insulin resistance (IR) index value decreased at 12 weeks compared with baseline in the LD. The consumption of legumes may accelerate weight loss accompanied by regulation of adiponectin and 8-epi-PGF $2 \alpha$ in obese subjects. In particular, legumes seemed to induce significant changes in BMI by increasing adiponectin in females. Additionally, increases in plasma adiponectin due to greater substantial weight loss may be related to the improvement in IR.

Key words: Legumes: Body weight: Adiponectin: 8-epi-PGF $2 \alpha$ : Insulin resistance

Obesity is one of the most severe health issues ${ }^{(1,2)}$. It is a significant risk factor for many metabolic diseases, such as CVD, diabetes and certain types of cancer, and it is associated with high mortality. The prevalence of obesity, including severe obesity, is increasing worldwide, and interest in dietary and lifestyle guidelines for the prevention and treatment of obesity is growing ${ }^{(3)}$.

Legumes are nutritionally recognised for their high protein content and soluble fibre ${ }^{(4)}$. Diets rich in legumes are beneficial because legumes contain thirty different polyphenols that modulate nitric oxide-dependent arterial, immune and inflammatory functions ${ }^{(5)}$. Legumes also contain some precursors of bioactive peptides that play essential roles in metabolic pathways ${ }^{(6)}$. The effects of dietary fibre on serum lipoproteins received much attention, but other biofunctional components in legumes are likely involved in their potential bioactivity ${ }^{(7)}$

There are numerous studies on dietary soya protein and lipid profiles, and some studies suggested that soya intake reduced CVD risk and oxidative stress via an antioxidant capacity ${ }^{(8,9)}$
Beneficial effects of non-soya legumes were also observed ${ }^{(10,11)}$. The impact of legume consumption on weight reduction ${ }^{(12)}$ and favourable outcomes for type II diabetes ${ }^{(13,14)}$ are well reported. However, limited clinical trials evaluated the effects of legumes on weight control, and the results are controversial ${ }^{(15)}$.

Adiponectin is an adipocytokine derived from adipose tissue that is closely related to obesity-related metabolic dysfunction because it plays a vital role in the regulation of lipid and glucose metabolism. Antioxidant-mediated adiponectin regulation by transcription factors, such as PPAR- $\gamma$ and sterol regulatory element-binding protein ${ }^{(16-18)}$, may be the potential mechanism of the favourable effects of legume consumption on weight control.

Legumes are rich in nutrients that have antioxidant effects. The link between the reduction in oxidative stress levels and weight loss was elucidated in several studies ${ }^{(19,20)}$. 8-Epi-PGF $2 \alpha$ is a secondary end product of peroxidation, and it is excreted in a stable form in urine ${ }^{(21,22)}$. It is a reliable indicator of oxidative

Abbreviations: HOMA, homoeostasis model assessment; hs-CRP, high-sensitivity C-reactive protein; IR, insulin resistance; LD, legume-enriched diet; UD, usual diet.

* Corresponding author: Minjoo Kim, email minjookim@hnu.kr 
stress, and an increased urinary level of 8-epi-PGF $2 \alpha$ was observed in subjects with abnormal glycaemic control or high oxidation states ${ }^{(23)}$. However, to our knowledge, no studies used 8-epi-PGF $F_{2 \alpha}$ as an index of oxidative stress to examine the antioxidant effects of legume consumption.

The present study performed a 12 -week nutritional intervention to determine whether a diet enriched in legumes was associated with weight loss via the regulation of adiponectin and 8-epi-PGF ${ }_{2 \alpha}$ in obese subjects. We also determined the mechanism of the effects of dietary legume consumption on weight loss.

\section{Methods}

\section{Subjects and study design}

A 12-week weight reduction programme was performed by the National Leading Research Laboratory of Clinical Nutrigenetics/ Nutrigenomics at Yonsei University. Subjects with a BMI of $25 \mathrm{~kg} / \mathrm{m}^{2}$ or higher based on the Asia-Pacific guideline ${ }^{(24)}$ were recruited at the National Health Insurance Corporation Ilsan Hospital in Goyang, Korea between March 2011 and December 2012. People with a history of type 2 diabetes, CVD, thyroid disorders, liver or kidney disease or psychiatric problems and/or using any medications (e.g. antihypertensive, lipid-lowering, antiplatelet and antidiabetic) were excluded. Paper-based informed consent forms were obtained from all participants. The Institutional Review Board of Yonsei University approved the study protocol, which complied with the Declaration of Helsinki. The present study is also registered at ClinicalTrials.gov under NCT04392882 (retrospectively registered 19 May 2020).

The sample size was determined using the $G *$ Power programme (Franz Faul) with an effect size of $0 \cdot 3$, statistical power of $80 \%$, a two-sided significance level of 0.05 and an allocation ratio of 1.4 . The calculation of the effect size was based on a previous clinical trial ${ }^{(25)}$ that showed significant changes in total body fat mass (\%) with a 12-week legume-based diet. Considering a $10 \%$ dropout rate, 400 participants were enrolled. The enrolled subjects ( $n$ 400) were randomly assigned to the legume-enriched diet (LD) group ( $n$ 168) or usual diet (UD) group ( $n$ 232) using a random number sequence generated in Microsoft Office Excel. The researchers were blinded until the intervention and assessment outcomes were collected. However, the registered dietitians were not blinded to manage the participants' compliance.

\section{Weight loss protocol and energy intake}

One week before starting the 12-week programme, the enrolled subjects provided their UD information on a semiquantitative FFQ and 24-h recall method. Registered dietitians gave written and verbal instructions on completing a 3-d (two weekdays and one weekend day) dietary record. Individually planned diets were programmed for each subject based on their obtained nutritional data and referring to the Korean RDA (Korean Nutrition Society). The LD group was assigned to replace onethird of their refined rice intake with legumes as a carbohydrate source. We recommended beans, especially black soya beans, but the legume carbohydrate sources were not limited to black soya beans. The UD group was advised to consume their UD.
Both groups were advised to increase their vegetable intake to at least six units (30-70 g/unit) per day to ensure a sufficient dietary fibre intake. The subjects were instructed to perform physical activity consisting of a regular 30-min walk after dinner each day.

A dietician interviewed participants biweekly via telephone to monitor compliance. Participants were asked whether they were following the programme well, including their dietary intake and physical activity. Standardised 3-d (two weekdays and one weekend day) dietary records were obtained from each participant at baseline and the 12-week follow-up visit. Dietary energy values and nutrient content were calculated using the Computer-Aided Nutritional Analysis Programme (CAN-pro 2.0; Korean Nutrition Society) based on the 3-d food records. A standardised physical activity record was also completed on the same day as the dietary record. The total energy expenditure of the subjects was calculated by multiplying the activity coefficient by the basal energy expenditure from the Harris-Benedict equation.

\section{Anthropometric parameters, blood pressure and blood collection}

All parameters were measured twice, at baseline (week 0) and follow-up (week 12). Body weights and heights were measured to calculate the BMI $\left(\mathrm{kg} / \mathrm{m}^{2}\right)$. Blood pressure was measured using an automatic BP monitor (TM-2654; A\&D) after resting. After a fasting period of $12 \mathrm{~h}$, venous blood specimens were collected in EDTA-treated or untreated tubes. Separated plasma and serum were stored at $-70^{\circ} \mathrm{C}$ until use in further analyses.

\section{Serum glucose, insulin, lipid profiles, high-sensitivity C-reactive protein and urinary 8 -epi-PGF $2 \alpha$}

Detailed information about the assessments of levels of fasting glucose, insulin and serum high-sensitivity C-reactive protein (hs-CRP) is described in our previous study ${ }^{(26)}$. Analyses of TAG and total cholesterol levels were performed using a Hitachi 7150 Autoanalyzer (Hitachi Ltd.). HDL-cholesterol was separated from apoB-containing lipoproteins using dextran sulphate-Mg and measured enzymatically. LDL-cholesterol was estimated using the Friedewald formula in subjects with a serum TAG level $\leq 400 \mathrm{mg} / \mathrm{dl}$. 8-Epi-PGF $2 \alpha$ was measured in urine using an enzyme immunoassay (BIOXYTECH urinary 8-epi-PGF ${ }_{2 \alpha}$ TM assay kit; OXIS International Inc.).

\section{Insulin resistance and adiponectin}

Insulin resistance (IR) was assessed using the homoeostasis model assessment (HOMA) formula: (fasting insulin $(\mu \mathrm{IU} / \mathrm{ml}) \times$ fasting glucose $(\mathrm{mmol} / \mathrm{l})) / 22 \cdot 5$. The plasma adiponectin concentration was measured using an enzyme immunoassay (Human Adiponectin ELISA kit; B-Bridge International Inc.) and Victor2 (Perkin Elmer Life Sciences).

\section{Statistical analyses}

We performed statistical analyses using SPSS ver 25.0 (SPSS Inc.). Before statistical analyses, the skewed variables were logarithmically transformed. To evaluate the differences in clinical variable 
levels between the two groups, independent $t$ tests were used. For analysing differences between baseline/12-week follow-up time scales, paired $t$ tests were used. Pearson's correlation coefficients were used to investigate the relationships between variables over time.

\section{Results}

Among the enrolled subjects $(n=400)$, eight from the LD group and nine from the UD group dropped out during the intervention period for personal reasons or poor compliance, leaving 383 subjects.

\section{Clinical characteristics and nutrient intake before and after dietary intervention}

There were no significant differences in the clinical characteristics or nutrient intake between the two groups at baseline. At 12 weeks, weight $(P<0 \cdot 001)$, BMI $(P<0 \cdot 001)$, systolic blood pressure $(P=0.037)$ and diastolic blood pressure $(P=0.001)$ decreased compared with baseline in the LD group. Based on the daily nutrient intake results, energy intake $(P<0 \cdot 001)$ and carbohydrate intake $(P<0.001)$ were reduced, and total energy expenditure $(P<0.001)$ and the intake of protein $(P=0.027)$, fat $(P<0.001)$ and fibre $(P=0.003)$ were increased in the LD group at 12 weeks compared with baseline. These patterns, except total energy expenditure $(P<0.001)$ and fibre intake $(P=0.037)$, were not present in the UD group at 12 weeks. Weight, BMI, energy, carbohydrate and fat intake showed significant differences at 12 weeks between the two groups and were different from baseline in both groups, but systolic blood pressure was significant only at 12 weeks (Table 1).

\section{Serum glucose, insulin, lipid profiles, high-sensitivity} C-reactive protein and 8-epi-PGF $2 \alpha$

At baseline, the two groups exhibited no significant differences in levels of serum glucose, insulin, hs-CRP, or 8-epi-PGF 2 $_{2 \alpha}$ or lipid profiles. The LD subjects showed significantly decreased levels of glucose $(P<0.001)$, insulin $(P<0.001)$ and 8 -epi-PGF $2 \alpha$ $(P=0.021)$, and the level of HDL-cholesterol $(P<0.001)$ increased dramatically after the intervention (Table 2 ). There were no significant changes in the UD subjects. The levels of HDL-cholesterol $(P=0.003)$, glucose $(P=0.039)$ and insulin $(P<0.001)$ were significantly different at the end of the 12 -week intervention between the two groups. The insulin level $(P=0.020)$ was significantly different between the two groups, and 8-epi-PGF ${ }_{2 \alpha}$ tended to differ after the 12-week intervention period (Table 2).

\section{TAG, homoeostasis model assessment-insulin resistance} index and adiponectin

No significant between-group differences were found at baseline in TAG or adiponectin levels or the HOMA-IR index values. After the 12 -week intervention, the TAG levels $(P<0 \cdot 001)$ and HOMA-IR index values $(P<0.001)$ decreased significantly, and the adiponectin levels $(P<0 \cdot 001)$ increased compared with baseline in the LD group. The UD group exhibited no differences (Fig. 1). The HOMA-IR index value was significantly different at
12 weeks between the LD and UD groups $(P<0.001)$ and changed from baseline $(P=0 \cdot 016)$. The change in the level of adiponectin was significantly different at 12 weeks $(P<0.001)$ and differed from baseline $(P<0 \cdot 001)$. The level of TAG only tended to differ between the two groups at the end of the 12-week follow-up (Fig. 1).

\section{Correlations between changes in BMI, HDL-cholesterol, homoeostasis model assessment-insulin resistance index and adiponectin}

Changes $(\Delta)$ in BMI and the HDL-cholesterol level $(r=-0 \cdot 146$, $P=0.004)$ and BMI and the adiponectin level negatively correlated $(r=-0.314, P<0.001)$ in a linear manner in all subjects (Fig. 2). $\triangle$ BMI and $\triangle$ HOMA-IR index values showed a positive correlation $(r=0.154, P=0.003)$. A negative correlation between $\Delta$ BMI and $\Delta$ adiponectin $(r=-0 \cdot 271, P=0 \cdot 003)$ was observed only in the LD group. $\Delta \mathrm{BMI}$ and $\Delta$ HDL-cholesterol negatively correlated in the UD group $(r=0 \cdot 161, P=0 \cdot 016)$, and $\Delta$ BMI tended to be positively associated with the $\Delta$ HOMA-IR index value and negatively correlated with $\Delta$ adiponectin (Fig. 2).

\section{The relationships between adiponectin, high-sensitivity C-reactive protein, 8-epi-PGF $2 \alpha$, anthropometric biomarkers and biochemical markers before and after the 12-week intervention}

The adiponectin level negatively correlated with weight $(r=-0 \cdot 150, \quad P=0 \cdot 009)$, the levels of TAG $(r=-0 \cdot 202$, $P<0.001)$, glucose $(r=-0 \cdot 140, P=0 \cdot 015)$ and insulin $(r=$ $-0 \cdot 115, P=0 \cdot 048)$, the HOMA-IR index value $(r=-0 \cdot 150$, $P=0 \cdot 010)$ and the hs-CRP level $(r=-0 \cdot 129, P=0 \cdot 026)$ in all subjects at baseline and positively correlated with the levels of HDLcholesterol $(r=0.162, P<0.001)$ and LDL-cholesterol $(r=0.162$, $P=0.005)$. The level of adiponectin negatively correlated with the levels of TAG $(r=-0.349, P<0 \cdot 001)$, HDL-cholesterol $(r=0 \cdot 189, P=0.042)$ and insulin $(r=-0 \cdot 236, P=0 \cdot 012)$, and the HOMA-IR index value $(r=-0 \cdot 215, P=0 \cdot 022)$ in the LD group at 12 weeks. However, the adiponectin level negatively correlated with the levels of TAG and glucose $(r=-0 \cdot 193$, $P=0.029 ; r=0 \cdot 204, P=0 \cdot 021$, respectively) and positively correlated with the level of HDL-cholesterol $(r=0.352$, $P<0.001)$ in the UD group at 12 weeks. The insulin level $(r=0.191, P=0.019)$, HOMA-IR index value $(r=0.199$, $P=0.015)$ and 8 -epi-PGF $2 \alpha$ level $(r=0.431, P<0.001)$ positively correlated with the TAG level in the LD group at 12 weeks. These trends were similar in the two groups at 12 weeks. The 8-epi$\mathrm{PGF}_{2 \alpha}$ level positively correlated with the levels of glucose and insulin and the HOMA-IR index value $(r=0.264$, $P=0.002 ; r=0.219, P=0.010 ; r=0.264, P=0.002$, respectively) in the LD group at 12 weeks, and these relationships were also observed in the UD group. Unexpectedly, there were no significant correlations between the adiponectin, hs-CRP and 8-epi$\mathrm{PGF}_{2 \alpha}$ levels in either group, except for a negative correlation between the levels of $\Delta$ hs-CRP and $\Delta 8$-epi-PGF ${ }_{2 \alpha}(r=-0 \cdot 172$, $P=0.032$ ) in the UD group (online Supplementary Fig. S1). 
Table 1. Clinical characteristics and macronutrient indices at baseline and after the 12-week dietary intervention period of the participants (Mean values with their standard errors of the mean)

\begin{tabular}{|c|c|c|c|c|c|}
\hline & \multicolumn{2}{|c|}{ Legume-enriched diet $(n=160)$} & \multicolumn{2}{|c|}{ Usual diet $(n=223)$} & \multirow[b]{2}{*}{$P$} \\
\hline & Mean & SEM & Mean & SEM & \\
\hline Male/female (\%) & $42 \cdot 5 / 57 \cdot 5$ & & $41 \cdot 7 / 58 \cdot 3$ & & 0.876 \\
\hline Age (year) & $49 \cdot 4$ & $1 \cdot 12$ & 48.5 & 0.85 & 0.530 \\
\hline \multicolumn{6}{|l|}{ Weight (kg) } \\
\hline Before & 74.5 & 0.85 & $74 \cdot 1$ & 0.69 & 0.758 \\
\hline After & 71.6 & $0.85^{\star \star *}$ & $74 \cdot 0$ & 0.68 & 0.029 \\
\hline Change & $-2 \cdot 87$ & 0.21 & -0.17 & 0.11 & $<0.001$ \\
\hline \multicolumn{6}{|l|}{ BMI $\left(\mathrm{kg} / \mathrm{m}^{2}\right)$} \\
\hline Before & $27 \cdot 6$ & 0.21 & $27 \cdot 4$ & 0.16 & 0.329 \\
\hline After & $26 \cdot 5$ & $0 \cdot 21^{\star \star \star}$ & $27 \cdot 3$ & $0 \cdot 16$ & 0.003 \\
\hline Change & -1.09 & 0.08 & -0.06 & 0.04 & $<0.001$ \\
\hline \multicolumn{6}{|c|}{ Systolic blood pressure (mmHg) } \\
\hline Before & $125 \cdot 2$ & $1 \cdot 30$ & $128 \cdot 6$ & $1 \cdot 21$ & 0.067 \\
\hline After & $122 \cdot 8$ & $1 \cdot 23^{*}$ & $128 \cdot 3$ & 1.05 & 0.001 \\
\hline Change & $-2 \cdot 41$ & $1 \cdot 14$ & -0.21 & 0.98 & 0.145 \\
\hline \multicolumn{6}{|c|}{ Diastolic blood pressure (mmHg) } \\
\hline Before & 79.5 & 0.85 & 79.9 & 0.77 & 0.720 \\
\hline After & $76 \cdot 8$ & $0 \cdot 80^{* *}$ & 78.6 & 0.77 & 0.096 \\
\hline Change & $-2 \cdot 63$ & $0 \cdot 77$ & $-1 \cdot 29$ & 0.66 & 0.187 \\
\hline \multicolumn{6}{|c|}{ Total energy expenditure (kcal/d) } \\
\hline Before & $2218 \cdot 5$ & $29 \cdot 6$ & $2192 \cdot 1$ & 23.4 & 0.479 \\
\hline After & $2308 \cdot 1$ & $31 \cdot 2^{\star \star \star}$ & $2265 \cdot 3$ & $24 \cdot 2^{\star \star \star}$ & 0.273 \\
\hline Change & $89 \cdot 6$ & $12 \cdot 8$ & 73.3 & $12 \cdot 3$ & 0.366 \\
\hline \multicolumn{6}{|c|}{ Estimates of daily nutrient intake } \\
\hline \multicolumn{6}{|c|}{ Energy intake (kcal) } \\
\hline Before & $2389 \cdot 5$ & $27 \cdot 9$ & $2380 \cdot 0$ & $29 \cdot 8$ & 0.824 \\
\hline After & $2147 \cdot 1$ & $39 \cdot 1^{\star \star *}$ & $2333 \cdot 2$ & $30 \cdot 7$ & $<0.001$ \\
\hline Change & -242.4 & $42 \cdot 5$ & $-52 \cdot 1$ & 34.6 & 0.001 \\
\hline \multicolumn{6}{|c|}{ Carbohydrate (\%) } \\
\hline Before & $60 \cdot 1$ & 0.47 & $60 \cdot 6$ & 0.32 & 0.363 \\
\hline After & $57 \cdot 7$ & $0.41^{\star * \star}$ & 59.9 & 0.40 & $<0.001$ \\
\hline Change & -2.35 & 0.52 & -0.69 & 0.51 & 0.027 \\
\hline \multicolumn{6}{|l|}{ Protein (\%) } \\
\hline Before & 20.5 & 0.39 & $20 \cdot 3$ & 0.25 & 0.699 \\
\hline After & 21.5 & $0.38^{*}$ & $21 \cdot 3$ & 0.51 & 0.820 \\
\hline Change & 0.99 & 0.44 & 1.03 & 0.56 & 0.968 \\
\hline \multicolumn{6}{|l|}{ Fat $(\%)$} \\
\hline Before & $19 \cdot 5$ & 0.41 & $19 \cdot 7$ & 0.27 & 0.729 \\
\hline After & $22 \cdot 0$ & $0 \cdot 29^{\star \star \star}$ & $19 \cdot 7$ & 0.21 & $<0.001$ \\
\hline Change & 2.47 & 0.43 & -0.04 & 0.32 & $<0.001$ \\
\hline \multicolumn{6}{|l|}{ Fibre $(\mathrm{g})$} \\
\hline Before & $11 \cdot 8$ & 0.52 & $12 \cdot 1$ & 0.60 & 0.176 \\
\hline After† & 14.5 & $0.69^{* *}$ & $14 \cdot 1$ & $0.65^{*}$ & 0.120 \\
\hline Change & $2 \cdot 77$ & 0.72 & 2.05 & 0.58 & 0.431 \\
\hline
\end{tabular}

${ }^{\star} P<0.05,{ }^{* \star} P<0.01,{ }^{* \star *} P<0.001$ compared with baseline values in each diet group as tested using paired $t$ test. $P$-values derived from independent $t$ test.

$\dagger$ Tested using logarithmic transformation.

Correlations between changes in major indicators in the legume-enriched diet group by sex

The results of the between-group and within-group comparisons in the LD group by sex (males and females in the LD group) revealed a similar trend as all subjects (data not shown). However, notable differences between the two groups were found in the correlation analysis between the changes in the values of the major indicators. Among the changed values in females in the LD group, $\Delta$ BMI positively correlated with $\Delta$ weight $(r=0.996, P<0.001), \Delta$ WHR $(r=0.399, P<0.001)$, and $\Delta$ HDL $(r=0.217, P=0.038)$ and negatively correlated with $\Delta$ glucose $(r=-0 \cdot 215, P=0.004), \Delta$ adiponectin $(r=-0.298$, $P=0.01)$ and $\Delta 8$-epi-PGF $\mathrm{PG}_{2 \alpha}(r=-0.229, P=0.039)$. However, $\Delta \mathrm{BMI}$ in males in the LD group only showed correlations with
$\Delta$ WHR $(r=0.544, P<0.001)$ and $\Delta$ systolic blood pressure $(r=-0.257, P=0.036) . \Delta$ Adiponectin negatively correlated with $\Delta \mathrm{WT}, \Delta \mathrm{TAG}$ and $\Delta \mathrm{LDL}(r=-0.289, P=0.013 ; r=$ $-0.324, P=0.005 ; \quad r=-0.391, P=0.001$, respectively) in females in the LD group (Fig. 3).

\section{Discussion}

The present study demonstrated that a 12-week LD may improve clinical variables in obese subjects. After 12 weeks, the LD group had significant reductions in body weight and BMI. Conversely, significant differences were not observed in the UD group who consumed the UD with increased vegetables. Our results partially confirm some clinical studies ${ }^{(27)}$. 
Table 2. Effects of dietary intervention on lipid profiles and levels of glucose, insulin, hs-CRP and 8-epi-PGF ${ }_{2 \alpha}$ at baseline and 12-week follow-up (Mean values with their standard errors of the mean)

\begin{tabular}{|c|c|c|c|c|c|}
\hline & \multicolumn{2}{|c|}{$\begin{array}{l}\text { Legume-enriched } \\
\text { diet }(n=160)\end{array}$} & \multicolumn{2}{|c|}{$\begin{array}{l}\text { Usual diet } \\
(n=223)\end{array}$} & \multirow[b]{2}{*}{$P$} \\
\hline & Mean & SEM & Mean & SEM & \\
\hline \multicolumn{6}{|c|}{ LDL-cholesterol (mg/dl) } \\
\hline Before $\dagger$ & 111.0 & $2 \cdot 44$ & $114 \cdot 6$ & $2 \cdot 60$ & 0.918 \\
\hline After† & $108 \cdot 0$ & $2 \cdot 61$ & $113 \cdot 1$ & 2.50 & 0.196 \\
\hline Change & -2.99 & 2.03 & -1.50 & $2 \cdot 17$ & 0.616 \\
\hline \multicolumn{6}{|c|}{ HDL-cholesterol (mg/dl) } \\
\hline Before† & $45 \cdot 6$ & 0.98 & $43 \cdot 7$ & 0.78 & 0.165 \\
\hline After† & $48 \cdot 1$ & $0.88^{* *}$ & $44 \cdot 8$ & 0.80 & 0.003 \\
\hline Change & 2.48 & 0.76 & 1.55 & 0.63 & 0.342 \\
\hline \multicolumn{6}{|c|}{ Glucose (mg/dl) } \\
\hline Before $\dagger$ & $97 \cdot 4$ & 1.82 & $99 \cdot 3$ & 1.78 & 0.531 \\
\hline After† & $93 \cdot 8$ & $2 \cdot 65^{\star *}$ & 97.9 & 1.99 & 0.039 \\
\hline Change & -3.59 & 1.94 & -1.41 & 1.63 & 0.388 \\
\hline \multicolumn{6}{|c|}{ Insulin $(\mu \mathrm{IU} / \mathrm{ml})$} \\
\hline Before† & $11 \cdot 8$ & 0.54 & $10 \cdot 8$ & 0.36 & 0.446 \\
\hline After† & $9 \cdot 17$ & $1.07^{\star *}$ & $10 \cdot 6$ & 0.52 & $<0.001$ \\
\hline Change & -2.58 & 0.96 & -0.17 & 0.53 & 0.020 \\
\hline \multicolumn{6}{|c|}{ NEFA $(\mu \mathrm{Eq} / \mathrm{l})$} \\
\hline Before $†$ & 532.4 & $1 \cdot 8 \cdot 0$ & 524.9 & $17 \cdot 3$ & 0.572 \\
\hline After† & 507.5 & $20 \cdot 1$ & 503.0 & $16 \cdot 3$ & 0.952 \\
\hline Change & -24.8 & $18 \cdot 8$ & -21.9 & $17 \cdot 7$ & 0.911 \\
\hline \multicolumn{6}{|c|}{ hs-CRP (mg/dl) } \\
\hline Before & 1.97 & 0.08 & 2.09 & 0.09 & 0.707 \\
\hline After† & 1.89 & 0.09 & 2.09 & 0.11 & 0.854 \\
\hline Change & -0.19 & 0.07 & 0.00 & 0.10 & 0.132 \\
\hline \multicolumn{6}{|c|}{ 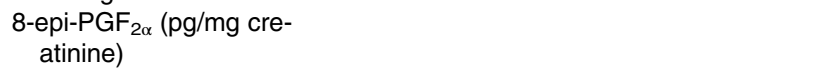 } \\
\hline Before $†$ & 1330.5 & 81.9 & $1346 \cdot 4$ & $66 \cdot 7$ & 0.504 \\
\hline After† & $1216 \cdot 6$ & $80 \cdot 7^{*}$ & $1299 \cdot 2$ & $65 \cdot 5$ & 0.077 \\
\hline Change & $-56 \cdot 3$ & $75 \cdot 7$ & $-36 \cdot 7$ & 65.9 & 0.844 \\
\hline
\end{tabular}

hs-CRP, high-sensitivity C-reactive protein.

${ }^{\star} P<0.05,{ }^{\star \star} P<0.001$ compared with baseline values in each diet group as tested using paired $t$ test. $P$-values derived from independent $t$ test.

† Tested using logarithmic transformation.

Papanikolaou and Fulgoni ${ }^{(28)}$ reported a relationship between bean consumption and obesity risk in approximately 8000 adult participants in the National Health and Nutrition Examination Survey (NHANES) 1999-2002 using 24-h dietary recall data. They found that people who consumed various beans or baked beans showed significantly lower body weight than people who had not consumed beans. The odds of being obese (BMI $>30 \mathrm{~kg} / \mathrm{m}^{2}$ ) were also significantly lower in people who consumed a variety of beans and baked beans $(\mathrm{OR}=0 \cdot 78$ and $0 \cdot 77$, respectively). However, Venn et al. showed a slightly different result. The incorporation of pulses and whole-grain foods into a weight loss programme resulted in an essential reduction in waist circumference compared with the control diet, but weight loss was not different between groups ${ }^{(29)}$.

After a 12-week intervention, energy intake (kcal), \% carbohydrate intake and \% fat intake were significantly different between the two groups in the present study. According to the Korea Health Statistics 2011: Korea National Health and Nutrition Examination Survey (KNHANES V-2), city populations consume approximately $19 \cdot 6 \%$ of total energy content from fat. Based on these data, our results showed significant differences between the LD and UD groups after the intervention $(22.0 \quad(\operatorname{sem} 0 \cdot 29) \quad v \cdot 19 \cdot 7(\operatorname{sem} \pm 0 \cdot 21))$. Because we instructed the LD group to replace one-third of a rice bowl with legumes, a dramatic decrease in the \% carbohydrate was present. Replacing one-third of a bowl of rice per meal per day results in a $418.4 \mathrm{~kJ}(100 \mathrm{kcal})$ deficit because a bowl of rice has $1255.2 \mathrm{~kJ}$ (300 kcal) according to the food composition tables from the Rural Development Administration (8th Ed., 2011) of $\mathrm{Korea}^{(30)}$. Therefore, the increase in \% fat observed in the LD group was due to the changes in the total energy intake (kcal), \% carbohydrate and \% protein, and it does not mean an absolute increase in fat intake by the LD group.

Notably, we demonstrated that a decrease in BMI correlated with an increase in the adiponectin level in obese subjects. Our results showed that plasma adiponectin levels negatively correlated with body weight, the HOMA-IR index value and the level of hs-CRP and positively correlated with the level of HDLcholesterol, which are consistent with previous studies ${ }^{(31,32)}$. Several studies reported that weight loss in massively obese subjects was associated with increases in serum adiponectin concentration ${ }^{(33,34)}$. Yannakoulia et al. noted that whole-grain intake was associated with high adiponectin levels. In a crosssectional study of 220 healthy Mediterranean women, adherence to a dietary pattern characterised by a high consumption of legumes, whole-grain cereals and low-fat dairy products was positively associated with adiponectin levels after controlling for potential confounders ${ }^{(35)}$. The LD group in our study also exhibited a significant decrease in the HOMA-IR index value and significant increase in adiponectin levels after the 12-week intervention. These data suggest that a more substantial amount of weight loss with legume consumption increased plasma adiponectin, which may control insulin and glucose metabolism.

Our sex-stratification analysis revealed that $\Delta$ adiponectin increased significantly in females in the LD group compared with females in the UD group. However, no significant difference was found between males from the LD and UD groups. $\Delta$ BMI was substantially larger in females than males in the LD group. $\triangle$ BMI showed a negative correlation with $\Delta$ adiponectin only in females in the LD group. Because sex hormones play a critical role in adipose tissue activity ${ }^{(36)}$, adiponectin generally exists at a lower level in males than females. This phenomenon is also closely related to fat, especially visceral fat ${ }^{(37,38)}$. Bidulescu et al. ${ }^{(38)}$ demonstrated an inverse relationship between abdominal visceral adipose tissue and serum adiponectin levels in African American females, but this association was not observed in males. Collectively, our results suggest that an LD helps reduce visceral and subcutaneous fat, especially in females. However, further research is needed to clarify the type of fat affected by legume consumption.

The significant decrease in 8-epi-PGF $2 \alpha$ levels in the LD group may result from a meaningful legume effect in mitigating oxidative stress ${ }^{(39)}$. Accumulated fat induces the production of reactive oxygen species and lipid peroxidation byproducts. Therefore, the considerable weight loss of the LD group may underlie the significant change in the 8-epi-PGF $2 \alpha$ level. The 8-epi$\mathrm{PGF}_{2 \alpha}$ level positively correlated with the levels of glucose and insulin and the HOMA-IR index value in the LD group at 12 weeks. Reactive oxygen species are closely related to a 
$\mathrm{TAG}^{\varnothing}$
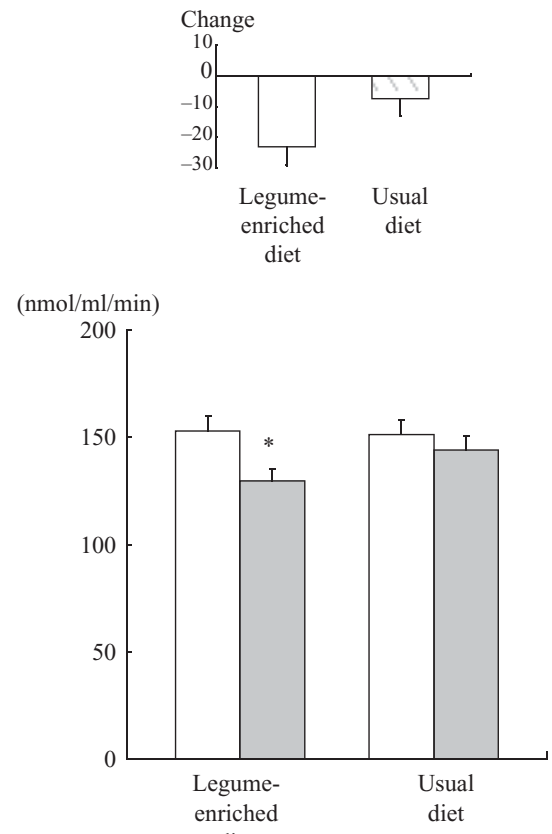

diet
HOMA-IR ${ }^{\varnothing}$
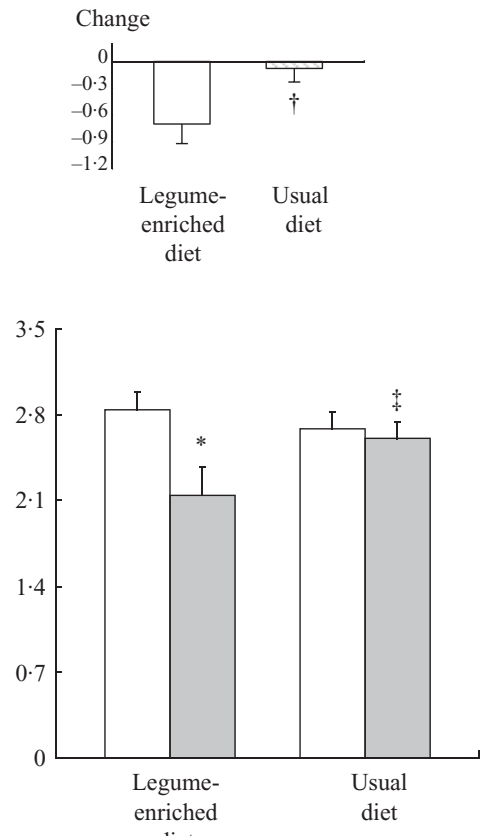

diet
Adiponectin $^{\phi}$
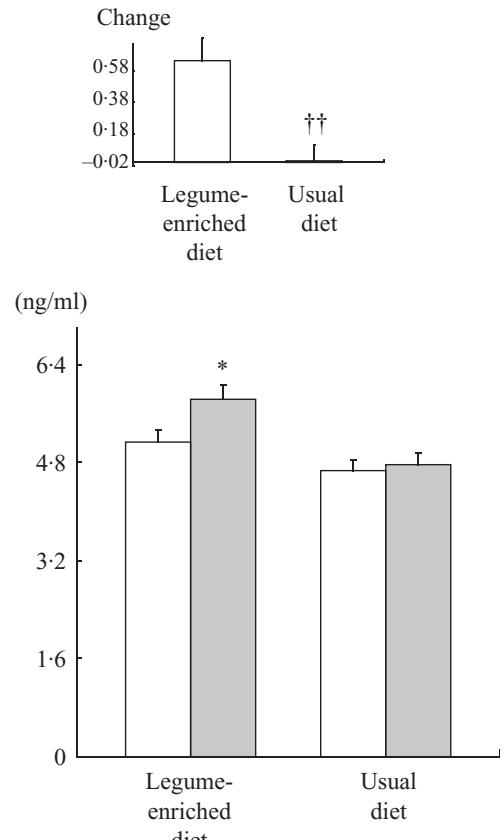

diet

Fig. 1. Effects of legume consumption on the levels of TAG and adiponectin and the HOMA-IR index value. Mean \pm SEM. ${ }^{\S}$ Tested using log transformation. ${ }^{\star} P<0.001$ compared with baseline values in each group as tested using paired $t$ test. $P$-values derived from independent $t$ test. $P^{\prime}=$ after adjusting for baseline value. ${ }^{\ddagger} P<0.001$ comparison between two groups at the 12-week follow-up and ${ }^{\dagger} P<0.05,{ }^{\dagger \dagger} P<0.001$ comparison between two groups at changed values tested using independent $t$ test. HOMA-IR, homoeostasis model assessment-insulin resistance. $\square$, baseline; $\square, 12$-week.
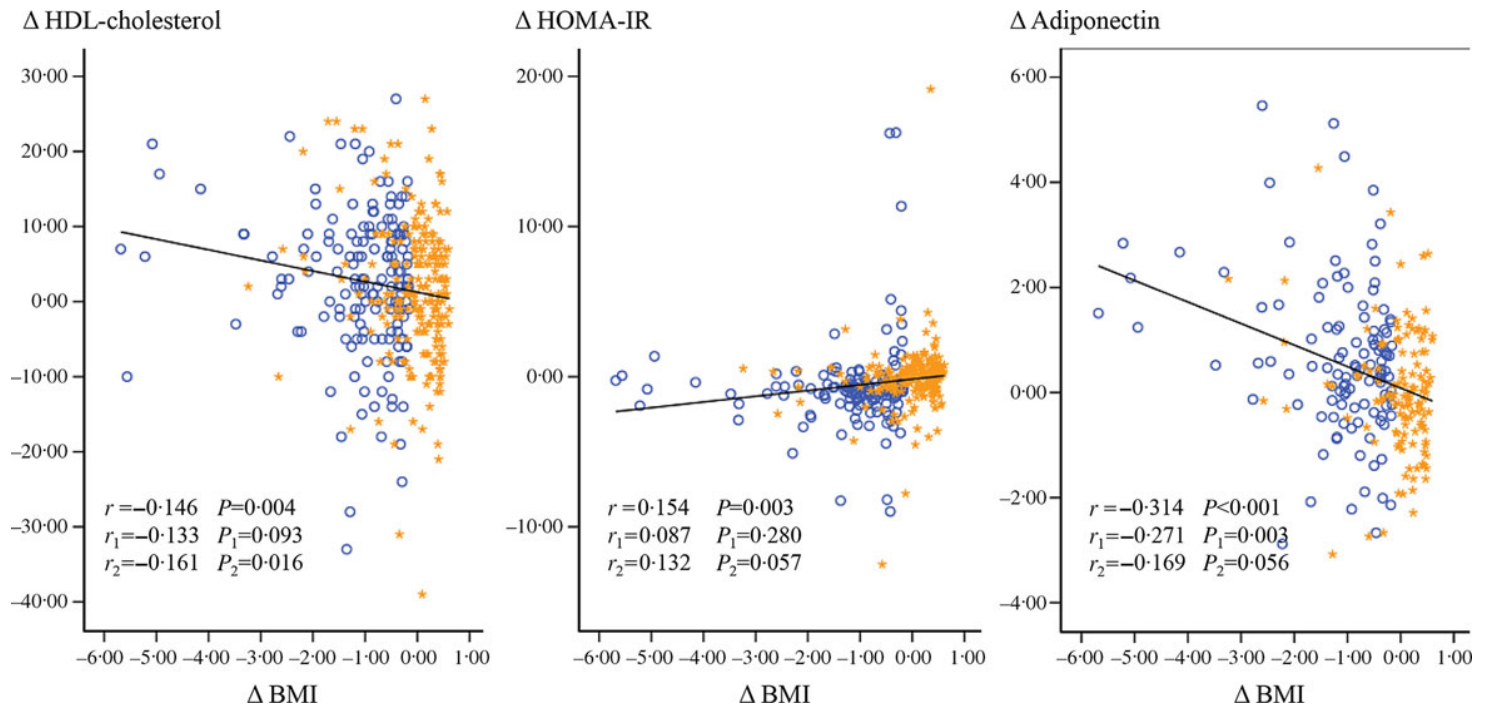

Fig. 2. Correlations between changes (difference from baseline) in BMI, the HOMA-IR index value, and the levels of HDL-cholesterol and adiponectin in 383 subjects. $r=$ Pearson's correlation coefficients in all subjects. $r_{1}=$ correlation coefficients in the LD group. $r_{2}=$ correlation coefficients in the UD group. HOMA-IR, homoeostasis model assessment-insulin resistance. $\bigcirc$, legume-enriched diet; $\star$, usual diet.

proinflammatory state with increasing inflammatory cytokine secretion and macrophage infiltration. Therefore, our findings suggest that legume consumption, in addition to weight loss, may help prevent chronic disease by controlling inflammatory conditions.

Several limitations of this work need to be explained. The first is that participants' dietary intake information was collected from self-reports. However, measurement errors from self-reported nutritional intake and lifestyle variables are relatively small ${ }^{(40)}$. Second, the control group established in our study was not sufficient. Further intervention studies with a control group in which one-third of refined rice intake is replaced with foods other than legumes are essential to clarify the effect of the LD. Alternatively, intervention studies are needed to compare groups consuming 
(a)

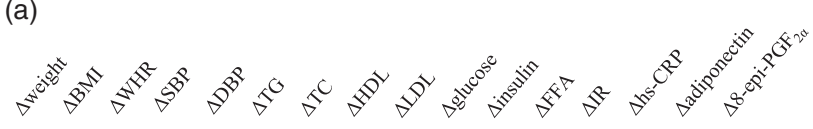

(b)

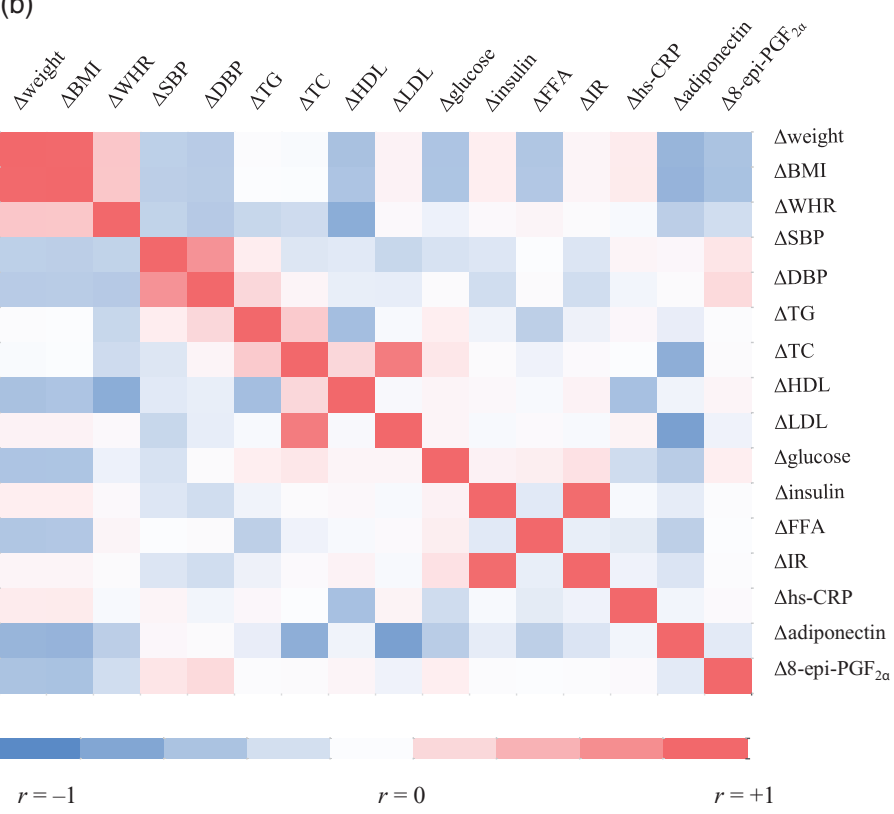

Fig. 3. Correlations between changes (difference from baseline) in major indicators in the legume-enriched diet group by sex. (a) Male with legume-enriched diet. (b) Female with legume-enriched diet. $r=$ Pearson's correlation coefficients. Red represents a positive correlation, and blue represents a negative correlation.

legumes with groups without legume supplementation. Third, it is difficult to clarify the causal relationships of the observed changes in adiponectin, 8-epi-PGF $\mathrm{PF}_{2 \alpha}$ and weight loss. The improvements in adiponectin and 8 -epi-PGF $2 \alpha$ might be directly affected by weight loss and might not be caused by legume consumption. However, several studies that shown that legumes could cause favourable metabolic changes, irrespective of weight loss ${ }^{(41,42)}$, have indicated that legumes itself improve adiponectin and 8-epi-PGF $\mathrm{PF}_{2 \alpha}$, similar to our findings. These major metabolic changes that appear to result from legume intake have been shown to be associated with weight loss. However, the dose-dependent effects of legume consumption were not considered in the present study, so it is difficult to conclude that the observed metabolic changes were directly caused by legume consumption. Thus, we suggest the possibility that the weight loss induced by LD confers health benefits such as regulating the levels of adiponectin and 8-epi-PGF $2 \alpha$. Notably, the consumption of legumes induced significant changes in adiponectin levels in females. Unexpectedly, legume antioxidant-mediated adiponectin regulation, which may help with weight reduction, could not fully be elucidated in the present study because a direct correlation was not observed between the levels of adiponectin and 8-epi-PGF $\mathrm{PG}_{2 \alpha}$. We also suggest that the increases in plasma adiponectin induced by more considerable weight loss with LD supplementation may be related to a greater activation of IR. Further studies are needed to confirm the exact mechanism of legume weight reduction based on this evidence.

\section{Declarations}

Ethics approval and consent to participate: The Institutional Review Board of Yonsei University approved the study protocol, which was performed in compliance with the Helsinki Declaration.

Consent for publication: Paper-based informed consent forms, stored in a document system after obtaining the necessary signatures, were used to record the intent and willingness to participate in the research.

Availability of data and materials: The datasets generated and/or analysed during the present study are available from the corresponding author on reasonable request.

\section{Acknowledgements}

The authors thank the research volunteers who participated in the studies described in this article.

This research was supported by the Basic Science Research Program through the National Research Foundation of Korea funded by the Ministry of Science and ICT (2012M3A9C4048762 and NRF-2017R1C1B2007195) and Ministry of Education (NRF2019R1I1A2A01061731).

Y. H., A. R. K., J. H. L. and M. K. analysed the data. J. H. L. and M. K. developed the study protocol and design. M. K. wrote the original draft. Y. H. and M. K. edited the manuscript. All of the authors read, commented on and contributed to the manuscript. J. H. L. and M. K. provided research funding and developed the study protocol. M. K. is the guarantor of this work, has full access to all data in the study and takes responsibility for the integrity of the data and the accuracy of the data analysis.

The authors declare that they have no competing interests.

\section{Supplementary material}

For supplementary material referred to in this article, please visit https://doi.org/10.1017/S0007114521000970 


\section{References}

1. Chooi YC, Ding C \& Magkos F (2019) The epidemiology of obesity. Metabolism 92, 6-10.

2. Agha M \& Agha R (2017) The rising prevalence of obesity: part a: impact on public health. Int J Surg Oncol 2, e17.

3. Swinburn BA, Caterson I, Seidell JC, et al. (2004) Diet, nutrition and the prevention of excess weight gain and obesity. Public Health Nutr 7, 123-146.

4. Kim HJ, Choi EJ, Kim HS, et al. (2019) Germinated soy germ extract ameliorates obesity through beige fat oxidation. Food Funct 10, 836-848.

5. Llorach R, Favaria C, Alonsoa D, et al. (2019) Comparative metabolite fingerprinting of legumes using LC-MS-based untargeted metabolomics. Food Res Int 126, 108666.

6. Malika B \& Myriern L-S (2013) Nutritional quality of legumes, and their role in cardiometabolic risk prevention: a review. JMed Food 16, 185-198.

7. Ruscica M, Pavanello C, Gandini S, et al. (2018) Effect of soy on metabolic syndrome and cardiovascular risk factors: a randomized controlled trial. Eur J Nutr 57, 499-511.

8. Jenkins DJ, Kendall CW, Vidgen E, et al. (2000) Effect of soybased breakfast cereal on blood lipids and oxidized low-density lipoprotein. Metabolism 49, 1496-1500.

9. Wiseman H, O'Reilly JD, Adlercreutz H, et al. (2000) Isoflavone phytoestrogens consumed in soy decrease F(2)-isoprostane concentrations and increase resistance of low-density lipoprotein to oxidation in humans. Am J Clin Nutr 72, 395-400.

10. Salehi-Abargouei A, Saraf-Bank S \& Bellissimo N (2015) Effects of non-soy legume consumption on C-reactive protein: a systemic review and meta-analysis. Nutrition 31, 631-639.

11. Bazzano LA, Thompson AM, Tees MT, et al. (2011) Non-soy legume consumption lowers cholesterol levels: a meta-analysis of randomized controlled trials. Nutr Metabo Cardiovas Dis 21, 94-103.

12. Jang EH, Moon JS, Ko JH, et al. (2008) Novel black soy peptides with antiobesity effects: activation of leptin-like signaling and AMP-activated protein kinase. Int J Obes 32, 1161-1170.

13. Azadbakht L \& Esmaillzadeh A (2009) Soy-protein consumption and kidney-related biomarkers among type 2 diabetics: a crossover, randomized clinical trial. J Ren Nutr 19, 479-486.

14. Azadbakht L, Atabak S \& Esmaillzadeh A (2008) Soy protein intake, cardiorenal indices, and C-reactive protein in type 2 diabetes with nephropathy: a longitudinal randomized clinical trial. Diabetes Care 31, 648-654.

15. Alizadeh M, Daneghian S, Ghaffari A, et al. (2011) The effect of hypocaloric diet enriched in legumes with or without L-arginine and selenium on anthropometric measures in central obese women. J Res Med Sci 15, 331-343.

16. Han SH, Quon MJ, Kim JA, et al. (2007) Adiponectin and cardiovascular disease: response to therapeutic interventions. $J \mathrm{Am}$ Coll Cardiol 49, 531-538.

17. Rayalam S, Della-Fera MA \& Baile CA (2008) Phytochemicals and regulation of the adipocyte life cycle. $J$ Nutr Biochem 19, 717-726.

18. Detopoulou P, Panagiotakos DB \& Chrysohoou C (2010) Dietary antioxidant capacity and concentration of adiponectin in apparently healthy adults: the ATTICA study. EurJ Clin Nutr 64, 161-168.

19. Choroma' nska B, My'sliwiec P, Łuba M, et al. (2020) Impact of weight loss on the total antioxidant/oxidant potential in patients with morbid obesity-a longitudinal study. Antioxidants 9, 376.

20. Navas-Carretero S, Cuervo M, Abete I, et al. (2011) Frequent consumption of selenium-enriched chicken meat by adults causes weight loss and maintains their antioxidant status. Biol Trace Elem Res 143, 8-19.
21. Milne GL, Musiek ES \& Morrow JD (2005) F2-isoprostanes as markers of oxidative stress in vivo: an overview. Biomarkers 10, S10-S23.

22. Morrow JD (2016) The isoprostanes-unique products of arachidonate peroxidation: their role as mediators of oxidant stress. Curr Pharm Des 12, 895-902.

23. Ahn HY, Kim M, Seo CR, et al. (2018) The effects of Jerusalem artichoke and fermented soybean powder mixture supplementation on blood glucose and oxidative stress in subjects with prediabetes or newly diagnosed type 2 diabetes. Nutr Diabetes $\mathbf{8}, 42$.

24. WHO Expert Consultation (2004) Appropriate body-mass index for Asian populations and its implications for policy and intervention strategies. Lancet 363, 157-163.

25. Allison DB, Gadbury G, Schwartz LG, et al. (2003) A novel soybased meal replacement formula for weight loss among obese individuals: a randomized controlled clinical trial. Eur J Clin Nutr 57, 514-522.

26. Kim M, Kim M, Yoo HJ, et al. (2018) Age-specific determinants of pulse wave velocity among metabolic syndrome components, inflammatory markers, oxidative stress. $J$ Atheroscler Thromb 25, 178-185.

27. Darmadi-Blackberry I, Wahlqvist ML, Kouris-Blazos A, et al. (2004) Legumes: the most important dietary predictor of survival in older people of different ethnicities. Asia Pac J Clin Nutr 13, 217-220.

28. Papanikolaou Y \& Fulgoni VL (2008) Bean consumption is associated with greater nutrient intake, reduced systolic blood pressure, lower body weight, and a smaller waist circumference in adults: results from the National Health and Nutrition Examination Survey 1999-2002. J Am Coll Nutr 27, 569-576.

29. Venn BJ, Perry T, Green TJ, et al. (2010) The effect of increasing consumption of pulses and whole-grains in obese people: a randomized controlled trial. J Am Coll Nutr 29, 365-372.

30. Kang M, Yoo HJ, Kim M, et al. (2018) Metabolomics identifies increases in the acylcarnitine profiles in the plasma of overweight subjects in response to mild weight loss: a randomized, controlled design study. Lipids Health Dis 17, 237.

31. Menzaghi C, Ercolino T, Di Paola R, et al. (2002) A haplotype at the adiponectin locus is associated with obesity and other features of the insulin resistance syndrome. Diabetes $\mathbf{5 1}$, 2306-2312.

32. Huang KC, Lue BH, Yen RF, et al. (2004) Plasma adiponectin levels and metabolic factors in nondiabetic adolescents. Obes Res 12, 119-124.

33. Yang WS, Lee WJ, Funahashi T, et al. (2001) Weight reduction increases plasma levels of an adipose-derived anti-inflammatory protein, adiponectin. J Clin Endocrinol Metab 86, 3815-3819.

34. Ng TWK, Watts GF, Barrett PH, et al. (2007) Effect of weight loss on LDL and HDL kinetics in the metabolic syndrome: associations with changes in plasma retinol-binding protein- 4 and adiponectin levels. Diabetes Care 30, 2945-2950.

35. Yannakoulia M, Yiannakouris N, Melistas L, et al. (2008) A dietary pattern characterized by high consumption of whole-grain cereals and low-fat dairy products and low consumption of refined cereals is positively associated with plasma adiponectin levels in healthy women. Metabolism 57, 824-830.

36. Selthofer-Relatić K, Radić R, Stupin A, et al. (2018) Leptin/ adiponectin ratio in overweight patients-gender differences. Diab Vasc Dis Res 15, 260-262.

37. Gariballa S, Alkaabi J, Yasin J, et al. (2019) Total adiponectin in overweight and obese subjects and its response to visceral fat loss. BMC Endocr Disord 19, 55. 
38. Bidulescu A, Liu J, Hickson DA, et al. (2013) Gender differences in the association of visceral and subcutaneous adiposity with adiponectin in African Americans: the Jackson Heart Study. BMC Cardiovasc Disord 13, 9.

39. Landi N, Pacifico S, Piccolella S, et al. (2015) Valle Agricola lentil, an unknown lentil (Lens culinaris Medik.) seed from southern Italy as a novel antioxidant and prebiotic source. Food Funct 6, 3155-3164.

40. Rimm EB, Giovannucci EL, Stampfer MJ, et al. (1992) Reproducibility and validity of an expanded self-administered semi-quantitative food frequency questionnaire among male health professionals. Am J Epidemiol 135, 1114-1126.

41. Mirmiran P, Hosseini S, Hosseinpour-Niazi S, et al. (2019) Legume consumption increase adiponectin concentrations among type 2 diabetic patients: a randomized crossover clinical trial. Endocrinol, Diabetes Nutr 66, 49-55.

42. Neuhouser ML, Schwarz Y, Wang C, et al. (2011) A low-glycemic load diet reduces serum C-Reactive Protein, modestly increases adiponectin in overweight, obese adults. I Nutr 142, 369-374. 